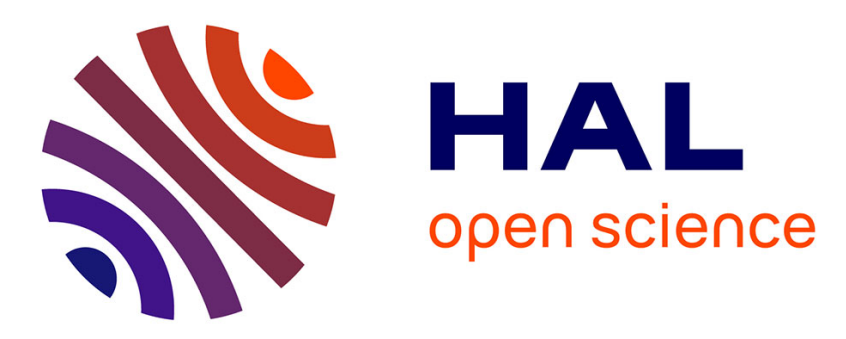

\title{
Flux Weakening of PMSM for Enhancing Torque Tracking
}

Jérémie Kreiss, Jean-François Trégouët, Romain Delpoux, Jean-Yves

Gauthier, Xuefang Lin-Shi

\section{- To cite this version:}

Jérémie Kreiss, Jean-François Trégouët, Romain Delpoux, Jean-Yves Gauthier, Xuefang Lin-Shi. Flux Weakening of PMSM for Enhancing Torque Tracking. ECC, Jun 2018, Limassol, Cyprus. pp.26652670, 10.23919/ECC.2018.8550573 . hal-02053183

\section{HAL Id: hal-02053183 \\ https://hal.science/hal-02053183}

Submitted on 1 Mar 2019

HAL is a multi-disciplinary open access archive for the deposit and dissemination of scientific research documents, whether they are published or not. The documents may come from teaching and research institutions in France or abroad, or from public or private research centers.
L'archive ouverte pluridisciplinaire HAL, est destinée au dépôt et à la diffusion de documents scientifiques de niveau recherche, publiés ou non, émanant des établissements d'enseignement et de recherche français ou étrangers, des laboratoires publics ou privés. 


\title{
Flux Weakening of PMSM for Enhancing Torque Tracking
}

\author{
Jérémie Kreiss $^{1}$, Jean-François Trégouët ${ }^{1}$, Romain Delpoux ${ }^{1}$, Jean-Yves Gauthier ${ }^{1}$ and Xuefang Lin-Shi ${ }^{1}$
}

\begin{abstract}
Permanent magnet synchronous motors associated to their drivers are subjected to both current and voltage saturations. Dealing with those limitations is the core of flux weakening problem which aims defining achievable operating conditions. In stark contrast with most of the literature focusing on the steady-state, this paper investigates how current and voltage constraints impact transient as well. In-depth analysis reveals that bounds affecting derivative of torque exerted by the machine are influenced by direct current. This suggests that by assigning proper value to this signal, one can shift torque limits beforehand in direction where high torque demand is expected to occur. Simulations suggest that this "preloading" strategy can indeed enhance torque tracking and help meeting the most demanding specifications. A comprehensive optimization framework for selecting the level of "preload" is also provided.
\end{abstract}

\section{INTRODUCTION}

Permanent Magnet Synchronous Motors (PMSMs) are widely used in industry and transportation. They are more robust than brushed DC motors and produce higher torque per volume than induction machines. Thanks to the coordinate transformation called direct-quadrature (d-q) transformation introduced in [1], control strategies for such motor has received considerable interest such as field oriented control. Among them, we find methods based on feedback linearization [2], sliding mode control [3], to cite a few.

For high speed applications, one of the primary limitation of PMSM is the boundedness of excitation control (i.e. voltage and current limitations). Indeed, internal electromotive force (EMF) rises in proportion to the motor speed to a speed limit for which voltage limit is reached. At the steady-state, fail to comply with current limitations, related to cooling capacity of the machine among others, might also damage the motor. However, adjusting direct current $i_{d}$ is possible by acting on voltages in order to reach higher speeds or produce larger torque. This is the so-called flux weakening operation (see [4], [5] or [6] to cite a few). The related problem, which aims computing the optimal electrical steady-state maximizing torque or speed under current and voltage constraints, has been extensively studied in the literature (see for instance [7], [8], [9]). The purpose of this paper is to analyze how those constraints impact transient as well.

Analysis of current and voltage constraints during transient reveals that bounds affecting $\dot{\tau}$, the derivative of torque produced by the machine, are influenced by direct current $i_{d}$. This suggests that by assigning proper value to $i_{d}$, one can shift limits of $\dot{\tau}$ beforehand in direction where high torque

\footnotetext{
${ }^{1}$ Every author is with Laboratoire Ampère, INSA Lyon, Université de Lyon, 20, Avenue Albert Einstein, 69100 Villeurbanne, France firstname. lastnamedinsa-lyon.fr
}

demand is expected to occur. Simulations suggest that this "preloading" strategy can indeed enhance torque tracking and help meeting the most demanding specifications.

Furthermore, it appears that "preload" might increase energy consumption. To order to compromise competing control goals of "preload" and losses minimization, an optimization framework is proposed which takes current and voltage constraints as well as stator resistance voltage droop into account. Its solution is computed analytically by way of geometrical considerations. Note that this last result gives, as a special case, analytical solution to the optimal steady-state selection problem of more classical statement like Torque Maximization [6].

The paper is organized as follows: After presenting considered model and associated control problem in Section II, description of "preloading" strategy is provided by Section III. In Section IV, selection of level of "preload" is formulated as an optimization whose analytical solution is given. In Section V, simulations are performed in order to illustrate the proposed approach. Finally, conclusions and perspectives are offered in Section VI.

Notations: We denote $y\left(t_{d}^{-}\right)\left(y\left(t_{d}^{+}\right)\right)$left (right) limit of time-varying signal $y(\cdot)$, i.e., limits of $y(t)$ when $t$ tends to $t_{d}$ with $t<t_{d}\left(t>t_{d}\right)$. The euclidean norm is denoted by $\|\cdot\|$.

\section{Problem statement}

As depicted on Fig. 1, the PMSM model considered in this paper breaks down into two parts: $(i)$ the electrical dynamic and (ii) the mechanical one.

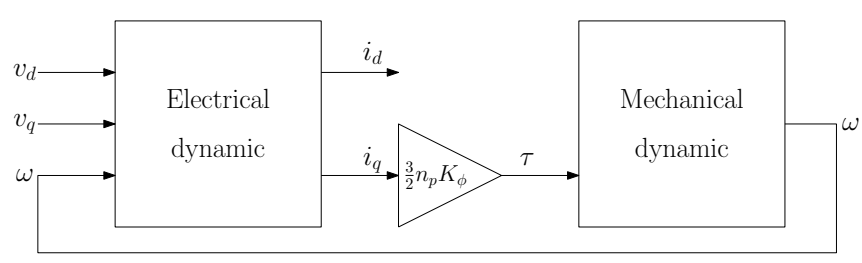

Fig. 1. Block diagram of PMSM dynamical model

\section{A. Electrical dynamic}

Consider the following state model of PMSMs electrical part in the $d-q$ reference frame (see [1]):

$$
L \frac{\mathrm{d}}{\mathrm{d} t} i=\left[\begin{array}{cc}
-R & n_{p} L \omega \\
-n_{p} L \omega & -R
\end{array}\right] i+v-\left[\begin{array}{c}
0 \\
K_{\phi} n_{p} \omega
\end{array}\right]
$$

where $v=\left[v_{d}, v_{q}\right]^{\top}$ and $i=\left[i_{d}, i_{q}\right]^{\top}$ gather respectively the $d$-axis, $q$-axis stator voltage and current, $L$ and $R$ are the stator inductance and resistance, $\omega$ is the mechanical angular velocity, $K_{\phi}$ is the permanent magnet flux linkage and $n_{p}$, the number of pole pairs. 
In this case, $v$ is the control vector whereas $i$ corresponds to state vector.

\section{B. Mechanical dynamic}

From the second Newton's law of motion in rotation, we obtain the model below for the mechanical part:

$$
J \frac{\mathrm{d}}{\mathrm{d} t} \omega=-f \omega+\tau-\tau_{\mathrm{ext}}
$$

where $J$ is the motor inertia, $f$ is the viscous frictional coefficient of the motor, $\tau_{\text {ext }}$ gathers external torque and $\tau$ is the electrical torque developed by the motor. This last signal has to be regarded as input of dynamics (2) while angular velocity $\omega$ is its state.

By relating $\tau$ and $i_{q}$, the following linear relationship:

$$
\tau=\frac{3}{2} n_{p} K_{\phi} i_{q}
$$

is the coupling term between electrical and mechanical dynamics.

\section{Torque tracking under constraints}

When tackling control problems related to mechanical variables, e.g., velocity or angular position tracking, disturbance rejection, etc., one typically implements modular control design for which reference torque $\tau^{\#}$ is computed by way of some high-level "mechanical control law". This signal then serves as a reference for low-level "electrical controller" driving current vector $i$. From (3), observe that $\tau$ depends on quadrature current $i_{q}$ solely which suggest that when tracking $\tau^{\#}$ one can ignore $i_{d}$. Yet, currents dynamics are coupled: From (1), derivative of $i_{q}$ is affected by $i_{d}$. However, as soon as $v$ can be freely selected, this coupling can be simply canceled out by substituting $v_{q}$ by $\hat{v}_{q}+n_{p} L \omega i_{d}$ so that derivative of $i_{q}$ does not depend on $i_{d}$ anymore and is controlled by new input $\hat{v}_{q}$.

Norm of $v$ is physically constrained, though, as this quantity cannot exceed some positive scalar $V_{\max }$ which depends on voltage source magnitude and control law driven the inverter. This implies that the following inequalities

$$
\|v\|=\sqrt{v_{d}^{2}+v_{q}^{2}} \leq V_{\max }
$$

holds at all time. Furthermore, $\|i\|$ is subjected to the following steady-state upper limits:

$$
\|i\|=\sqrt{i_{d}^{2}+i_{q}^{2}} \leq I_{\max }
$$

Overtaking this bounds, whose magnitude depends mainly on cooling capacity of the machine, might damage the motor.

Those two constraints might prevent from using change of variable involving $\hat{v}_{q}$. This is where direct current $i_{d}$ comes into play: When focusing on the steady-state, it comes out that decreasing $i_{d}$ can enlarge the set of achievable torque and speed. The problem of computing the optimal electrical steady-state for maximizing torque or speed under (4) and (5) has been extensively studied in the literature (see for instance [7], [8] or [9]). Usually, when system is away from saturation bounds, $i_{d}$ is left at 0 in order to minimize energy consumption. Negative value is assigned to this signal whenever voltage $v$ or current $i$ reach their limits.

The main contribution of this paper is to show that direct current $i_{d}$ can be used to improve transient reference torque tracking as well.

\section{CONTROLling $i_{d}$ TO ENHANCE TORQUE TRACKING}

Let us now investigate how inequalities (4) and (5) impose limits on mechanical input $\tau$ and, in turn, might prevent $\tau^{\#}$ from being properly tracked.

First note that (5) only restricts steady-state and, hence, can be violated during transient. As far as (4) is concerned, this constraint involves voltages and, in turn, imposes limitations on derivative of $\tau$. Indeed, by (1) and (3), one gets

$$
\begin{aligned}
\frac{\mathrm{d}}{\mathrm{d} t} \tau & =\frac{3}{2} n_{p} K_{\phi} \frac{\mathrm{d}}{\mathrm{d} t} i_{q} \\
& =\frac{3}{2 L} n_{p} K_{\phi}\left(-n_{p} \omega L i_{d}-R i_{q}+v_{q}-K_{\phi} n_{p} \omega\right)
\end{aligned}
$$

Considering the case where the system is initially at some equilibrium, let us compute maximum achievable $\dot{\tau}$ satisfying (4). From (1) and (2) and using continuity argument, it holds:

$$
i_{d}\left(t^{+}\right)=i_{d}\left(t^{-}\right), \quad i_{q}\left(t^{+}\right)=i_{q}\left(t^{-}\right), \quad \omega\left(t^{+}\right)=\omega\left(t^{-}\right),
$$

for any instant time $t$, see [10, Sec. 7.4]. As far as electrical inputs are concerned, discontinuity of $v$ is supposed to be achievable. Even at the electrical time scale, this hypothesis is indeed reasonable since $v$ is generated via Pulse Width Modulation (PWM) strategy driven by duty cycle which can (almost) instantaneously change value. This is equivalent to say that $v_{d}\left(t^{+}\right)$can be uncorrelated to $v_{d}\left(t^{-}\right)$, and the same fact applies to $v_{q}$. From (6) and the above discussion, it holds

$$
\begin{array}{r}
\frac{\mathrm{d}}{\mathrm{d} t} \tau\left(t^{+}\right)=\frac{3}{2 L} n_{p} K_{\phi}\left(-n_{p} \omega\left(t^{-}\right) L i_{d}\left(t^{-}\right)-R i_{q}\left(t^{-}\right)\right. \\
\left.+v_{q}\left(t^{+}\right)-K_{\phi} n_{p} \omega\left(t^{-}\right)\right)
\end{array}
$$

Expressing $i_{q}$ in terms of $\tau$ with (3) and replacing it in (8) leads to

$$
\begin{aligned}
& \dot{\tau}_{\min }\left(\omega\left(t^{-}\right), \tau\left(t^{-}\right), i_{d}\left(t^{-}\right)\right) \\
& \leq \dot{\tau}\left(t^{+}\right) \leq \\
& \quad \dot{\tau}_{\max }\left(\omega\left(t^{-}\right), \tau\left(t^{-}\right), i_{d}\left(t^{-}\right)\right)
\end{aligned}
$$

where

$$
\begin{array}{r}
\dot{\tau}_{\min }\left(\omega, \tau, i_{d}\right):=\psi(\omega) i_{d}+\gamma(\omega, \tau)-\frac{3}{2 L} n_{p} K_{\phi} V_{\max }, \\
\dot{\tau}_{\max }\left(\omega, \tau, i_{d}\right):=\psi(\omega) i_{d}+\gamma(\omega, \tau)+\frac{3}{2 L} n_{p} K_{\phi} V_{\max }, \\
\psi(\omega):=-\frac{3}{2} n_{p}^{2} K_{\phi} \omega, \quad \gamma(\omega, \tau):=-\frac{1}{L}\left(R \tau+\frac{3}{2} n_{p}^{2} K_{\phi}^{2} \omega\right),
\end{array}
$$

since largest magnitude of $v_{q}\left(t^{+}\right)$under (4) is $V_{\max }$ and is achieved for $v_{d}\left(t^{+}\right)=0$.

Given a mechanical equilibrium characterized by $\omega\left(t^{-}\right)$ and $\tau\left(t^{-}\right)$, the crucial point is that bounds of $\dot{\tau}\left(t^{+}\right)$depends on $i_{d}\left(t^{-}\right)$. As a result, if we can predict in same way, in which direction some torque of large magnitude will have to be exerted, bounds of $\dot{\tau}\left(t^{+}\right)$can be shifted in advance 
in the appropriate direction by controlling $i_{d}\left(t^{-}\right)$in order to enhance torque tracking. Such a goal can be indeed achieved since $\omega$ and $\tau$ do not uniquely define system equilibrium, provided that saturation limits are not reached.

The strategy described above and consisting in setting a non zero value to $i_{d}$ to shift bounds on derivative of $\tau$ will be coined "flux preloading" in the sequel. Note that this objective might be contradictory with minimization of $\|i\|$, a desirable objective for reducing energy consumption.

Let us now formulate the problem tackled in this paper.

Problem (Optimal flux preloading). Assign steady-state of PMSM compromising:

1) Maximization of ability of closed-loop system to response to abrupt torque demand by shifting bounds on $\dot{\tau}$

2) Minimization of input energy consumption, related to $\|i\|$;

and satisfying constraints (4) and (5).

Remark (Zero velocity case). Note that when $\omega=0$, neither $\dot{\tau}_{\min }$ nor $\dot{\tau}_{\text {max }}$ depend on $i_{d}$ since $\psi(0)=0$. As a result, the strategy is only applicable whenever considered mechanical steady-state involves non zero angular velocity.

\section{OPTIMAL FLUX PRELOADING}

This section aims formalizing problem stated in previous section into an optimization problem. For the sake of simplicity, let us focus on maximization of the upper bound of $\dot{\tau}$, since lower bound minimization can be handled in a similar way.

\section{A. Cost function}

Define the following cost function $\mathscr{J}: \mathbb{R}^{2} \rightarrow \mathbb{R}$

$$
\mathscr{J}\left(i_{d}, i_{q}\right)=\alpha\left(\frac{\|i\|}{I_{\max }}\right)^{2}-(1-\alpha) \frac{\dot{\tau}_{\max }\left(\omega, \tau, i_{d}\right)}{\left|\psi(\omega) I_{\max }\right|}
$$

parametrized by $\omega, \tau$ and $\alpha \in[0,1]$, the latter being a weighting variable compromising the two terms of $\mathscr{J}$. To understand the role played by those two terms, let us describe the two extreme cases related boundary interval of $\alpha$ :

- $\alpha=0$ is related to pure "flux preloading" which aims maximizing upper limit of $\dot{\tau}$ (or minimizing its opposite), regardless of energy consumption;

- $\alpha=1$ minimizes energy consumption, ignoring limits of $i$.

Note that both terms of $\mathscr{J}$ have been normalized via maximum norm of current vector $I_{\max }$ and $\psi(\omega)$.

This interpretation suggests that parameter $\alpha$ can be seen as a "responsivity" indicator of the controller: Large value of $\alpha$ should be used whenever abrupt variation of $\tau^{\#}$ is expected, requiring $\tau$ to grow very fast. The opposite case, corresponding to selection of low value of $\alpha$, indicates that smooth variation of $\tau^{\#}$ is expected, so that one can stick to energy consumption as the primary goal. In the case of mechanical disturbance rejection, a (slowly) time-varying signal can even be assigned to $\alpha$ and takes larger value when environnement is hostile than when it is safe.

\section{B. Optimization problem formulation}

Besides current and voltage inequalities given by (4) and (5), respectively, constraints that apply at the steady-state, characterized by some angular velocity $\omega$ and torque $\tau$, are simply:

$$
\frac{\mathrm{d}}{\mathrm{d} t} \omega=0, \quad \frac{\mathrm{d}}{\mathrm{d} t} i=\left[\begin{array}{l}
0 \\
0
\end{array}\right]
$$

This gives rise to the following optimization problem:

$$
\begin{aligned}
\min _{i_{d}, i_{q}}\left(\alpha \frac{i_{d}^{2}+i_{q}^{2}}{I_{\max }^{2}}-(1-\alpha) \frac{\dot{\tau}_{\max }\left(\omega, i_{d}\right)}{\left|\psi(\omega) I_{\max }\right|}\right) & \text { s.t. } \\
\left\|\left[\begin{array}{l}
i_{d} \\
i_{q}
\end{array}\right]\right\| & \leq I_{\max } \\
\left\|\left[\begin{array}{c}
0 \\
K_{\phi} n_{p} \omega
\end{array}\right]-\left[\begin{array}{cc}
-R & n_{p} L \omega \\
-n_{p} L \omega & -R
\end{array}\right]\left[\begin{array}{l}
i_{d} \\
i_{q}
\end{array}\right]\right\| & \leq V_{\max } \\
i_{q} & =\frac{2 \tau}{3 n_{p} K_{\phi}}
\end{aligned}
$$

where $\{\omega, \tau, \alpha\}$ acts as parameters set. Indeed, (11b) is nothing but current inequality (5) whereas (11c) corresponds to (4) after having been translated in terms of $i$ by zeroing derivatives of $i$ in (1). Equality (11d) readily derives from (3) and is related to torque to be exerted in order to compensate for frictional and external torques. Observe that this last constraint (11d) induces that $i_{d}$ is actually the only decision variable.

\section{Analytical solution of (11)}

This subsection derives analytical solution for problem (11) by way of geometrical considerations, rather than using Karush-Kuhn-Tucker optimality conditions. Specifically, constraints of (11) are depicted in the $\left(i_{d}, i_{q}\right)$-plan in hopes of better highlighting physical limitations and, hence, provide more informative discussion than "blind" construction of the Lagrangian.

1) Current saturation (11b) with (11d): An example about this saturation is depicted by Fig. 2. First note that (11d) sets the value of $i_{q}$ so that optimal solution belongs to the red horizontal line in $\left(i_{d}, i_{q}\right)$-plan satisfying (11d). Then remarking that $(11 \mathrm{~b})$ corresponds to a circle in $\left(i_{d}, i_{q}\right)$ plan centered at the origin, combination of (11b) and (11d) imposes that $i_{d}$ takes value within a segment:

$$
i_{d} \in\left[-i_{\text {sat }}, i_{\text {sat }}\right]
$$

where

$$
i_{\mathrm{sat}}=\sqrt{I_{\max }^{2}-\left(\frac{2 f \omega}{3 n_{p} K_{\phi}}\right)^{2}}
$$

2) Voltage saturation (11c) with (11d): As far as (11c) is concerned, let us first introduce the following change of variable

$$
\left[\begin{array}{c}
\tilde{i}_{d} \\
\tilde{i}_{q}
\end{array}\right]:=\left[\begin{array}{c}
i_{d} \\
i_{q}
\end{array}\right]+\kappa(\omega)\left[\begin{array}{c}
n_{p} \omega L \\
R
\end{array}\right]
$$

with

$$
\kappa(\omega):=\frac{K_{\phi} n_{p} \omega}{R^{2}+\left(n_{p} \omega L\right)^{2}}
$$




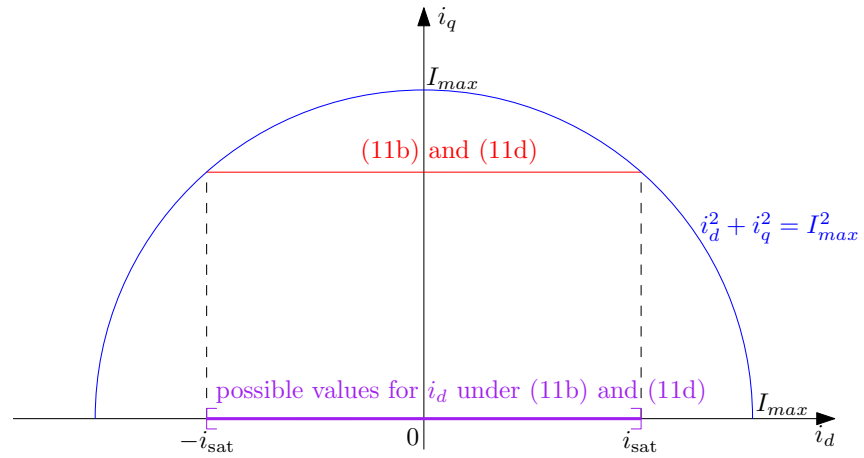

Fig. 2. Current limits (11b) in $\left(i_{d}, i_{q}\right)$-plan

so that (11c) becomes

$$
\begin{aligned}
& \left\|-\left[\begin{array}{cc}
-R & n_{p} L \omega \\
-n_{p} L \omega & -R
\end{array}\right]\left[\begin{array}{c}
\tilde{i}_{d} \\
\tilde{i}_{q}
\end{array}\right]\right\| \\
& =\sqrt{\left(-n_{p} \omega L \tilde{i}_{q}+R \tilde{i}_{d}\right)^{2}+\left(n_{p} \omega L \tilde{i}_{d}+R \tilde{i}_{q}\right)^{2}} \leq V_{\max }
\end{aligned}
$$

since

$$
\begin{aligned}
\kappa(\omega)\left[\begin{array}{cc}
-R & n_{p} L \omega \\
-n_{p} L \omega & -R
\end{array}\right]\left[\begin{array}{c}
n_{p} \omega L \\
R
\end{array}\right] & =-\kappa(\omega)\left[\begin{array}{c}
0 \\
\left(n_{p} L \omega\right)^{2}+R^{2}
\end{array}\right] \\
& =-\left[\begin{array}{c}
0 \\
K_{\phi} n_{p} \omega
\end{array}\right] .
\end{aligned}
$$

Expanding squared terms of (14) gives

$$
\tilde{i}_{d}^{2}+\tilde{i}_{q}^{2} \leq \frac{V_{\max }^{2}}{R^{2}+\left(n_{p} \omega L\right)^{2}}
$$

which corresponds, in $\left(i_{d}, i_{q}\right)$-plan, to equation of the disk centered at

$$
\left(-n_{p} \omega L \kappa(\omega),-R \kappa(\omega)\right)
$$

and of radius equals to

$$
r(\omega):=\frac{V_{\max }}{\sqrt{R^{2}+\left(n_{p} \omega L\right)^{2}}} .
$$

Thus, combining (11c) and (11d) leads to

$$
i_{d} \in\left[v_{\mathrm{sat}}^{-}, v_{\mathrm{sat}}^{+}\right]
$$

where

$$
\begin{aligned}
& v_{\mathrm{sat}}^{-}:=-\sqrt{r^{2}(\omega)-\left(\frac{2 f \omega}{3 n_{p} K_{\phi}}+R \kappa(\omega)\right)^{2}}-n_{p} \omega L \kappa(\omega) \\
& v_{\mathrm{sat}}^{+}:=\sqrt{r^{2}(\omega)-\left(\frac{2 f \omega}{3 n_{p} K_{\phi}}+R \kappa(\omega)\right)^{2}}-n_{p} \omega L \kappa(\omega) .
\end{aligned}
$$

As depicted by Fig. 3, $v_{\text {sat }}^{-}$and $v_{\text {sat }}^{+}$correspond to intersections between the line resulting from (11d) and boundary of disk (15).

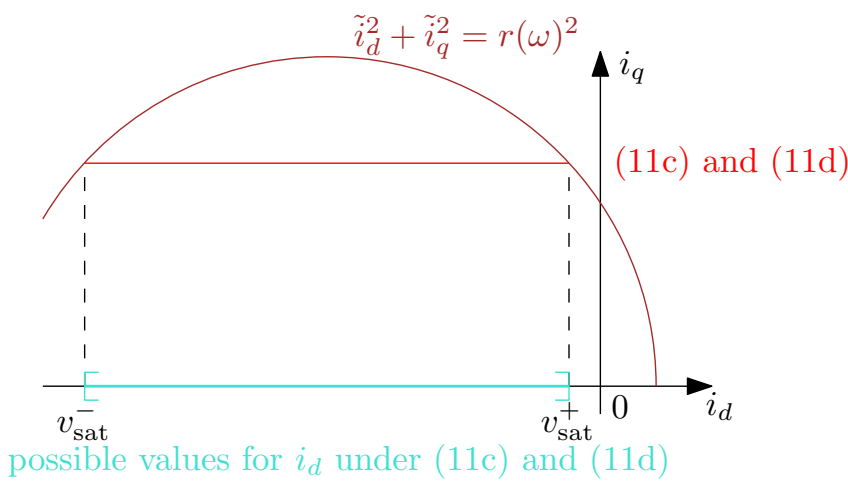

Fig. 3. Voltage limits (11c) in $\left(i_{d}, i_{q}\right)$-plan

3) Solution of the full problem: Combining (12) and (16) allows to reduces (11b), (11c) and (11d) to the simple relationship $i_{d} \in\left[\mathrm{sat}^{-}, \mathrm{sat}^{+}\right]$where

$$
\text { sat }^{-}:=\max \left\{-i_{\text {sat }}, v_{\text {sat }}^{-}\right\}, \quad \text { sat }^{+}:=\min \left\{i_{\text {sat }}, v_{\text {sat }}^{+}\right\} .
$$

as represented by Fig 4. As a result, optimal solution of (11) is given by

$$
i_{d}=\left\{\begin{array}{lr}
\text { sat }^{-}, & i_{d}^{*}<\mathrm{sat}^{-} \\
i_{d}^{*}, & \text { sat }-\leq i_{d}^{*} \leq \mathrm{sat}^{+} \\
\text {sat }^{-}, & i_{d}^{*}>\text { sat }^{+}
\end{array}\right.
$$

where $i_{d}^{*}$ refers to $i_{d}$ coordinate of the (unique) optimal solution $^{1}$ of simplified version of (11) which gets rid of inequality constraints (11b) and (11c):

$$
i_{d}^{*}:=\underset{i_{d}}{\arg \min } \mathscr{J}\left(i_{d}, \frac{2 \tau}{3 n_{p} K_{\phi}}\right) .
$$

Expression of $i_{d}^{*}$ derives by zeroing derivative of $\mathscr{J}$ with respect to $i_{d}$ :

$\frac{\partial}{\partial i_{d}} \mathscr{J}\left(i_{d}, \frac{2 \tau}{3 n_{p} K_{\phi}}\right)=0 \Leftrightarrow 2 \alpha \frac{i_{d}}{I_{\max }^{2}}-(1-\alpha) \frac{\psi(\omega)}{\left|\psi(\omega) I_{\max }\right|}=0$

which leads to

$$
i_{d}^{*}= \begin{cases}\frac{\alpha-1}{2 \alpha} I_{\max } \operatorname{sign}(\omega) & (\alpha>0) \\ +\infty & (\alpha=0 \wedge \omega>0) \\ -\infty & (\alpha=0 \wedge \omega<0)\end{cases}
$$

When $\alpha=0$, cost function $\mathscr{J}\left(i_{d}, 2 \tau /\left(3 n_{p} K_{\phi}\right)\right)$ is linear and, hence, unbounded from below. This is why infinity value is assigned to $i_{d}^{*}$, so that either $i_{d}^{*}<\mathrm{sat}^{-}$or $i_{d}^{*}>\mathrm{sat}^{+}$hold, inducing, by (17), that optimal solution $i_{d}$ lies on boundary of the feasible set.

Remark (Unfeasible cases). Depending on $\omega$ and $\tau$, problem (11) might be unfeasible. Fig. 4 graphically shows that such a situation occurs whenever the red line, related to (11d), lies above or below intersection of the two disks representing (11b) and (11c). In this case, inequality $\mathrm{sat}^{-}>\mathrm{sat}^{+}$hold. This gives a way to express critical value of $(\omega, \tau)$ by solving $\mathrm{sat}^{-}=\mathrm{sat}^{+}$, both terms depending on parameters $(\omega, \tau)$.

\footnotetext{
${ }^{1}$ Uniqueness indeed comes from strict convexity of $\mathscr{J}\left(\cdot, i_{q}\right)$ for any $i_{q}$ and $\alpha>0$, coming from linearity of $\dot{\tau}_{\max }$ with respect to $i_{d}$.
} 


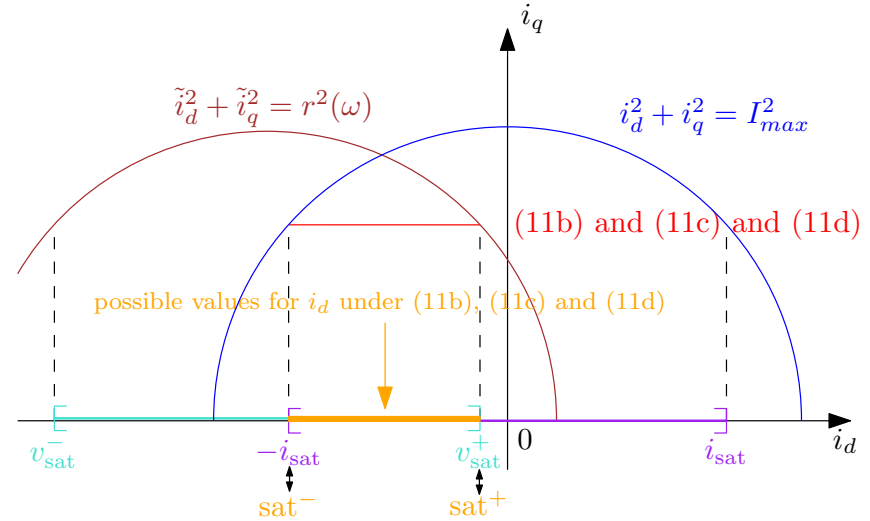

Fig. 4. Voltage and current limits (11b) and (11c) in $\left(i_{d}, i_{q}\right)$-plan

\section{Simulation Results}

Simulations are performed on open-loop model defined by (1) and (2) in order to highlight benefit of flux preloading for different values of $\omega, \tau$ and $\alpha$. Motor parameters are presented in Table I.

TABLE I

SYSTEM PARAMETERS

\begin{tabular}{lc}
\hline Pole pairs, $n_{p}$ & 5 \\
Stator resistance, $R$ & $0.55 \Omega$ \\
Stator inductance, $L$ & $4 e^{-4} \mathrm{H}$ \\
PM flux linnkage, $K_{\phi}$ & $0.0082 \mathrm{~Wb}$ \\
Viscous frictional coef., $f$ & $1.044 e^{-4} \mathrm{~kg} \cdot \mathrm{m}^{-1}$ \\
Inertia, $J$ & $2.2 e^{-6} \mathrm{~kg} \cdot \mathrm{m}^{2}$ \\
Voltage limitation, $V_{\max }$ & $12 \mathrm{~V}$ \\
Current limitation, $I_{\max }$ & $3 \mathrm{~A}$ \\
\hline
\end{tabular}

For each simulation running through $t=0$ to $t=t_{f}=100 \mu \mathrm{s}$, two distinct phases can be distinguished:

1) For $t \in\left[0, t_{d}[\right.$, system is at rest, at a mechanical equilibrium characterized by given $\omega$ and $\tau$;

2) For $t \in\left[t_{d}, t_{f}\right]$, electrical inputs are set to values that maximizes $\dot{\tau}\left(t_{d}^{+}\right)$under constraints (4), that is $v_{d}=0$ and $v_{q}=V_{\max }$ (see Section III).

Input signals are constant on each phase and transition occurs at $t_{d}=20 \mu \mathrm{s}$. During phase 1 , steady-state is further defined by selecting $i_{d}$ as solution of optimization problem (11). Different values of $\alpha$ are chosen in order to assess how this parameter impacts both phases.

A. Relevance of simulation environment for closed-loop system

In order to promote the simulation environment described above, let us consider the following realistic scenario where closed-loop strategy give rise to phases previously discussed.

Assume that velocity tracking is the foremost control goal. This corresponds to make the relative velocity $\widetilde{\omega}$, defined by

$$
\widetilde{\omega}:=\omega-\omega^{\#},
$$

as close to zero as possible. Here, $\omega^{\#}(\cdot)$ refers to timevarying reference to be tracked. Specifically, assume that this signal is piecewise constant and change value at $t=t_{d}$, while the system is initially at rest, i.e., $\widetilde{\omega}\left(t^{-}\right)=0$. Suppose, further, that mechanical controller delivering torque reference $\tau^{\#}$ is a proportional negative feedback of the form $-k \widetilde{\omega}$ with $k>0 .^{2}$ In this case, $\tau^{\#}$ will abruptly change value at $t=t_{d}$ since it holds

$\tau^{\#}\left(t_{d}^{+}\right)-\tau^{\#}\left(t_{d}^{-}\right)=-k\left(\widetilde{\omega}\left(t^{+}\right)-\widetilde{\omega}\left(t^{-}\right)\right) \stackrel{(7)}{=} k\left(\omega^{\#}\left(t^{+}\right)-\omega^{\#}\left(t^{-}\right)\right)$. so that $\omega^{\#}\left(t^{+}\right)>\omega^{\#}\left(t^{-}\right)$implies $\tau^{\#}\left(t_{d}^{+}\right)>\tau^{\#}\left(t_{d}^{-}\right)$indicating that $\tau$ has to be increased to drive $\widetilde{\omega}$ back to zero. Furthermore, if $k\left(\omega^{\#}\left(t^{+}\right)-\omega\left(t^{-}\right)\right)$is large, because $\omega^{\#}$ jump is wide or $k$ is selected to make the controller aggressive, the best closed-loop system can do it to produce the largest achievable control torque $\tau$ in the appropriate direction. This corresponds to increase $\tau$ as fast as possible by setting voltage inputs $v_{d}=0$ and $v_{q}=V_{\max }$ (see Section III). This is nothing but the simulation environment considered in this section.

\section{B. Definition of phase 1}

1) Case 1: $\omega=100 \mathrm{rad} . \mathrm{s}^{-1}$ and $\tau_{\text {ext }}=0$ : The lack of external torque induces that $\tau$ has only to compensate for frictional torque at the equilibrium so that $\tau=f \omega$. This allows to compute the following numerical values related to phase 1:

$$
\begin{array}{ll}
\text { - } \omega=100 \mathrm{rad}_{\mathrm{s}}^{-1} ; & \bullet i_{\mathrm{sat}}=2.995 \mathrm{~A} ; \\
\text { - } \tau=0.0104 \mathrm{~N} \cdot \mathrm{m} ; & \bullet v_{\mathrm{sa}}^{+}=16.967 \mathrm{~A} ; \\
\text { - } i_{q}=0.170 \mathrm{~A} ; & \bullet v_{\mathrm{sat}}^{-}=-21.755 \mathrm{~A} .
\end{array}
$$

which constraint $i_{d}$ to satisfy $-2.995 \mathrm{~A} \leq i_{d} \leq 2.995 \mathrm{~A}$.

2) Case 2: $\omega=300 \mathrm{rad} . \mathrm{s}^{-1}$ and $\tau_{\text {ext }}=0$ : In order to evaluate how rotor speed impacts the results, let us introduce second simulation setup for which larger value of $\omega$ is considered for phase 1 . Proceeding as before, new numerical values related to this phase are as follows:

$$
\begin{array}{ll}
\text { - } \omega=300 \mathrm{rad} . \mathrm{s}^{-1} ; & \bullet i_{\mathrm{sat}}=2.96 \mathrm{~A} ; \\
\text { - } \tau=-0.031 \mathrm{~N} . \mathrm{m} ; & \bullet v_{\mathrm{sat}}^{+}=-1.02 \mathrm{~A} ; \\
\text { - } i_{q}=0.51 \mathrm{~A} ; & -v_{\mathrm{sat}}=-21.26 \mathrm{~A}
\end{array}
$$

which constrain $i_{d}$ to satisfy $-2.96 \mathrm{~A} \leq i_{d} \leq-1.02 \mathrm{~A}$.

In contrast with the previous case, upper bound of $i_{d}$ is now induced by voltage saturation, i.e., $\mathrm{sat}^{+}=\mathrm{sat}_{v}^{+}$, and imposes negative direct current.

For both cases, $i_{d}$ is then computed as solution of problem (11) (given by (17)) for different $\alpha$. Results are provided by second column of Table II.

TABLE II

SOLUTION OF (11) AND DISSIPATED POWER

\begin{tabular}{|c|c|c|}
\hline \multicolumn{3}{|c|}{$\boldsymbol{\omega}=100$ rad.s $^{-1}$} \\
\hline$\alpha$ & $i_{d}(\mathrm{~A})$ & $P(\mathrm{~W})$ \\
\hline 1.00 & 0.000 & 0.024 \\
0.90 & -0.333 & 0.115 \\
0.75 & -1.000 & 0.849 \\
0.60 & -2.000 & 3.324 \\
0.50 & -2.995 & 7.425 \\
\hline
\end{tabular}

\begin{tabular}{|c|c|c|}
\hline \multicolumn{3}{|c|}{$\boldsymbol{\omega}=\mathbf{3 0 0}$ rad.s $^{-1}$} \\
\hline$\alpha$ & $i_{d}(\mathrm{~A})$ & $P(\mathrm{~W})$ \\
\hline 0.75 & -1.02 & 1.07 \\
0.70 & -1.29 & 1.58 \\
0.65 & -1.62 & 2.37 \\
0.60 & -2.00 & 3.51 \\
0.50 & -2.96 & 7.43 \\
\hline
\end{tabular}

Red values indicates that $\alpha$ is such that optimal value belongs to boundary of the constraints, i.e., $i_{d}$ equal either sat $^{-}$or $\mathrm{sat}^{+}$.

\footnotetext{
${ }^{2}$ Note that the same reasoning applies in the case where this controller additionally include integral part.
} 
Last column of Table II gives electrical power of resistive losses in electrical circuit during phase 1 and defined as follows

$$
P(i):=\frac{3}{2} R\left(i_{d}^{2}+i_{q}^{2}\right),
$$

according to [11, eq. (14)]. Recall that $\alpha$ aims parameterizing trade-off between energy consumption (large $\alpha$ ) and accuracy of torque tracking (low $\alpha$ ). This is in agreement with results of Table II since $P$ decreases as $\alpha$ takes larger value.

\section{Impacts of $\alpha$ on phase 2}

For the two simulation cases considered, let us investigate how phase 1, for which $\alpha$ serves as a parameter, impacts the first instant of phase 2. To this end, chronographs of $i_{d}$ and $\tau$ are depicted on Fig. 5 and Fig. 6, corresponding to $\omega=100$ rad. $\mathrm{s}^{-1}$ and $\omega=300$ rad. $\mathrm{s}^{-1}$, respectively.

On each figures, $i_{d}$ and $\tau$ curves are depicted using the same color for a given $\alpha$. It can be seen that the lower $i_{d}$ is (similarly the lower $\alpha$ is) in phase 1 (for $t \in[0,20[\mu \mathrm{s}$ ), the faster $\tau$ increases. This conclusion is obviously valid whenever selection of $\alpha$ does not induce a saturate value of $i_{d}$, corresponding to red values in previous table. It confirms that increasing $\alpha$ shift bounds of $\dot{\tau}$ in such a way that torque tracking can be enhanced under voltage saturation limits.

Finally, observe that preloading strategy is much more effective for large value of angular velocity. Indeed, as suggested by remark on Section III, bounds on $\dot{\tau}$ are influence by $i_{d}$ by way of term $\psi(\omega) i_{d}$ and $\psi$ is a linear function of its argument. As a result, large $\omega$ inducing strong "controllability" on $\psi(\omega) i_{d}$ and, in turn, on $\dot{\tau}_{\min }$ and $\dot{\tau}_{\max }$.
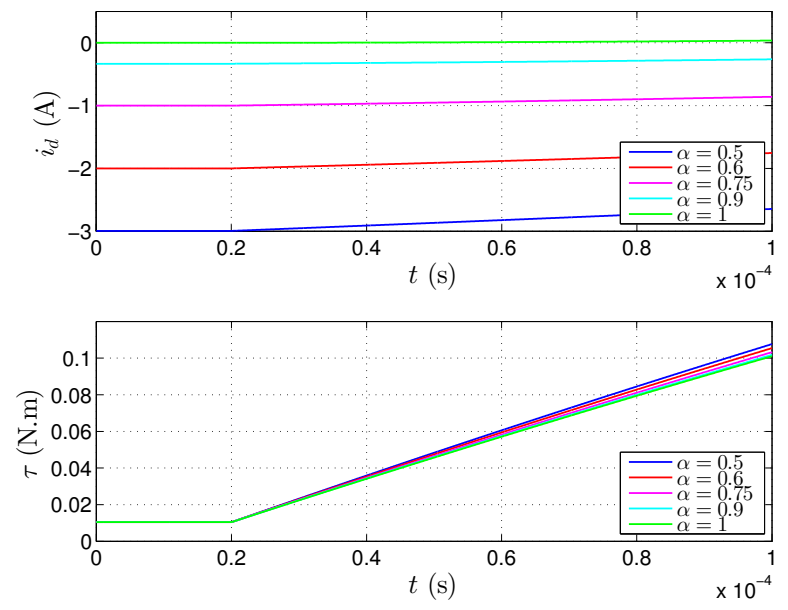

Fig. 5. Chronographs of $i_{d}(t)$ and $\tau(t)$ for $\omega=100 \mathrm{rad}_{\mathrm{s}} \mathrm{s}^{-1}$ and $\tau_{\mathrm{ext}}=0$

\section{CONCLUSION}

In this paper, it has been shown that torque limits of PMSM might be shifted during steady-state in contemplation of upcoming demanding transient. Numerical simulation demonstrates that this flux preloading strategy effectively enhance torque tracking. In order to compromise competing control goals of preloading and energy consumption, an optimization framework is proposed. It takes stator resistance as well as current and voltage constraints into account.
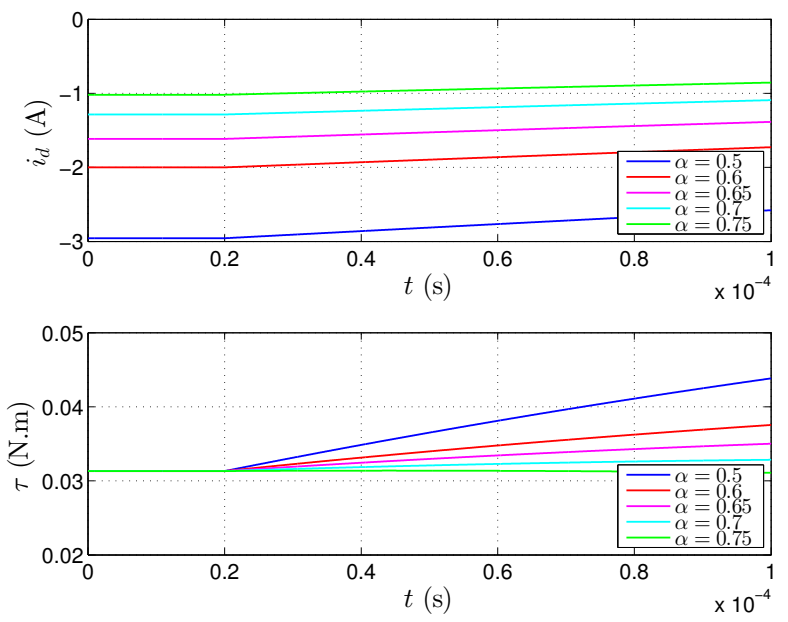

Fig. 6. Chronographs of $i_{d}(t)$ and $\tau(t)$ for $\omega=300 \mathrm{rad}^{-1}{ }^{-1}$ and $\tau_{\mathrm{ext}}=0$

This paper has to be regarded as a proof of concept. Going further would require to integrate flux preloading strategy into a feedback controller computing torque reference online and ensuring that current references are properly tracked, despites unknown perturbations and badly estimated parameters.

Allowing signal $\alpha$, parameterizing trade-off between energy consumption and responsivity, to be time-varying is also promising as it gives a way to adapt on-line closed-loop behavior to changing operating conditions.

\section{REFERENCES}

[1] R.-H. Park, "Two-reaction theory of synchronous machines generalized method of analysis-part i," Transactions of the American Institute of Electrical Engineers, vol. 48, no. 3, pp. 716-727, jul 1929.

[2] M. Bodson, J.-N. Chiasson, R.-T. Novotnak, and R.-B. Rekowski, "High performance nonlinear feedback control of a permanent magnet stepper motor," IEEE Transactions on Control Systems Technology, vol. 1 , no. 1 , pp. 5-14, 1993.

[3] M. Defoort, T. Floquet, A.-M. Kokosy, and W. Perruquetti, "A novel higher order sliding mode control scheme," Systems \& Control Letters, vol. 58, no. 2, pp. 102-108, 2009.

[4] D. Lu and N. C. Kar, "A review of flux-weakening control in permanent magnet synchronous machines," in 2010 IEEE Vehicle Power and Propulsion Conference, Sept 2010, pp. 1-6.

[5] M. Zordan, P. Vas, M. Rashed, S. Bolognani, and M. Zigliotto, "Fieldweakening in high-performance pmsm drives: a comparative analysis," in Conference Record of the 2000 IEEE Industry Applications Conference., vol. 3, 2000, pp. 1718-1724 vol.3.

[6] A. Verl and M. Bodson, "Torque Maximization for Permanent Magnet Synchronous Motors," IEEE Transactions on Control Systems Technology, vol. 6, no. 6, pp. 740-745, 1998.

[7] S. Bolognani, L. Peretti, and M. Zigliotto, "Online mtpa control strategy for dtc synchronous-reluctance-motor drives," IEEE Transactions on Power Electronics, vol. 26, no. 1, pp. 20-28, Jan 2011.

[8] M. Preindl and S. Bolognani, "Model predictive direct torque control with finite control set for pmsm drive systems, part 2: Field weakening operation," IEEE Transactions on Industrial Informatics, vol. 9, no. 2, pp. 648-657, May 2013.

[9] H. Eldeeb, C. M. Hackl, J. Kullick, and L. Horlbeck, "Analytical solutions for the optimal reference currents for mtpc/mtpa, mtpv and mtpf control of anisotropic synchronous machines," in 2017 IEEE International Electric Machines and Drives Conference (IEMDC), May 2017, pp. 1-6.

[10] E. A. Coddington and R. Carlson, Linear ordinary differential equations. SIAM, 1997.

[11] P. Pillay and R. Krishnan, "Modeling, simulation, and analysis of permanent-magnet motor drives. i. the permanent-magnet synchronous motor drive," IEEE Transactions on Industry Applications, vol. 25, no. 2, pp. 265-273, mar 1989. 